\title{
Pharmacogenomics as a risk mitigation strategy for chemotherapeutic cardiotoxicity
}

\author{
Brian C Jensen ${ }^{1,2, *}$ and Howard L McLeod ${ }^{1,3,4}$ \\ 1 UNC School of Medicine, Division of Cardiology, 160 Dental Circle, CB 7075, Chapel Hill, NC \\ 27599-7075, USA \\ 2UNC McAllister Heart Institute, Chapel Hill, NC, USA \\ ${ }^{3}$ Eshelman School of Pharmacy, Chapel Hill, NC, USA \\ 4 UNC Institute for Pharmacogenomics \& Individualized Therapy, Chapel Hill, NC, USA
}

\begin{abstract}
Damage to the heart can result from both traditional chemotherapeutic agents, such as doxorubicin, and newer 'targeted' therapies, such as trastuzumab. This chemotherapeutic cardiotoxicity is potentially life-threatening and necessitates limiting or discontinuing an otherwise-effective cancer treatment. Clinical strategies focus on surveillance rather than prevention, although there are no specific therapies for this highly morbid adverse effect. Current models for prospectively predicting risk of chemotherapeutic cardiotoxicity are limited. Cardiotoxicity can occur idiosyncratically in patients without obvious demographic risk factors, suggesting a genetically determined susceptibility, and candidate-gene studies have identified a limited number of variants that increase risk. In this commentary we indicate a need for more powerful means to identify risk prospectively, and suggest that broad pharmacogenomic approaches may be fruitful.
\end{abstract}

\section{Keywords}

anthracyclines; antineoplastic agents; cardiomyopathy; dilated; heart failure; pharmacogenetics

Cancer is a collection of diseases caused by the dysregulated proliferation of various cell types. Drugs that treat cancer either broadly inhibit cellular proliferation or specifically target proteins that are essential to expanding the population of abnormal cells. In contrast to many other tissue types, heart muscle has a very limited capacity for cellular proliferation. Its functional constituent cell type - the cardiomyocyte - is terminally differentiated and incapable of division. Thus, the development of cardiotoxicity in response to numerous chemotherapeutic agents is somewhat surprising from a biological perspective.

Nevertheless, patients who receive these agents are at increased risk of sustaining heart damage, which ranges from asymptomatic decrease in contractile function to overt heart

\footnotetext{
(C) 2013 Future Medicine Ltd

*Author for correspondence: bcjensen@med.unc.edu.

For reprint orders, please contact: reprints@ futuremedicine.com

Financial \& competing interests disclosure

HL McLeod received support from UL1 RR025747 and P01CA142538. BC Jensen received support from K08HL96836. The authors have no other relevant affiliations or financial involvement with any organization or entity with a financial interest in or financial conflict with the subject matter or materials discussed in the manuscript apart from those disclosed.

No writing assistance was utilized in the production of this manuscript.
} 
failure and death. In this article, we consider the cardiotoxicity associated with anthracyclines, a traditional class of antineoplastic agents, as well as trastuzumab and other newer drugs with a specific molecular target. Despite considerable efforts toward understanding the determinants of risk, clinicians remain unable to ascertain prospectively which patients will develop these life-threatening adverse reactions. We conclude that there is a pressing need to develop novel strategies to identify patients at high risk for chemotherapeutic cardiotoxicity.

\section{Anthracyclines: the past \& present of chemotherapeutic cardiotoxicity}

Doxorubicin (Adriamycin) and the related compounds daunorubicin and epirubicin are anthracycline antibiotics with broad antineoplastic activity, at least partially mediated by DNA intercalation and inhibition of topoisomerase II [1]. Their efficacy in the treatment of hematologic malignancies first was demonstrated in the 1960s [2,3], and doxorubicin in particular has been a mainstay of therapy for leukemia, lymphoma, breast cancer and numerous other malignancies for decades. The potency of anthracyclines was well illustrated in a meta-analysis published in 2005, which showed that use of an anthracyclinebased chemotherapeutic regimen for the treatment of breast cancer reduces the annual disease-related mortality by $38 \%$ in women younger than 50 and by $20 \%$ in women between 50 and 70 years of age [4].

The cardiotoxicity of this highly effective class of chemotherapy was not predicted in preclinical studies $[5,6]$ and initially was thought to be limited to children receiving especially high doses of the drug. Subsequently, however, the identification of fatal heart failure in multiple adults treated with ostensibly 'safe' doses of these drugs led some early investigators to suggest limiting their use to patients less than 40 years of age [7]. Further experience demonstrated a proportional increase in the risk of cardiotoxicity with higher cumulative dose [8] leading to a decrease in the incidence of this dreaded complication, but cardiomyopathy and heart failure continue to occur in both children and adults who receive doxorubicin [9]. Even low-dose regimens carry at least 5-15\% risk of cardiotoxicity [10,11], and clinical trial data likely underestimate the burden of cardiotoxicity owing to overly narrow definitions or limited follow-up intervals [12]. In fact, as many as $50 \%$ of patients who receive doxorubicin may develop subclinical cardiac damage [10,13]. Importantly, even asymptomatic left-ventricular dysfunction carries substantial risk of subsequent heart failure and death [14]. As survivorship and longitudinal follow-up intervals increase [15-17], the population of doxorubicin recipients ages and the associated cardiac morbidity and mortality can be expected to increase [18].

Despite decades of investigation, the mechanisms underlying doxorubicin-induced cardiotoxicity remain incompletely understood [19]. Ample experimental evidence (reviewed in [20,21]) demonstrate contributions to anthracycline-related cardiomyocyte death from both oxidative stress [22-26] and enhancement of proapoptotic signaling pathways [27-32], as well as failure of DNA-repair mechanisms and direct damage to the cardiac sarcomere [21]. Multiple clinical trials have demonstrated cardioprotection from the iron-chelating agent dexrazoxane $[33,34]$ and mouse models have suggested that disruptions in iron-handling pathways may predispose to doxorubicin-induced cardiotoxicity [35]. The limitations of our mechanistic understanding of this process are manifest in the lack of effective means to predict its occurrence. The most common surveillance strategies for the detection of incident cardiomyopathy consist of serial cardiac imaging using echocardiography or radionuclide angiography [36]. Although these measures are relatively sensitive and surveillance should be practiced universally, they may simply alert physicians to the presence of irreversible damage. Newer molecular imaging techniques may prove more sensitive for the detection of early damage, but there currently are no molecular means 
to prospectively and specifically identify patients at risk. Some centers also follow serial cardiac biomarkers, such as cardiac troponin and natriuretic peptides, in patients who receive anthracyclines. Although small studies suggest merit to this approach [37], there are no prospective data demonstrating the efficacy or cost-effectiveness of cardiac biomarker surveillance in mitigating risk of anthracycline-induced cardiotoxicity.

Management of anthracycline-induced cardiomyopathy consists of neurohormonal antagonism with ACE inhibitors and $\beta$-adrenergic receptor antagonists ( $\beta$-blockers), although patients often experience progressive heart failure despite these agents. This practice has been extrapolated from abundant evidence of decreased morbidity and mortality in heart failure patients treated with these neurohormonal antagonists, and likely is underused in chemotherapeutic cardiotoxicity [38]. Although dexrazoxane has demonstrated efficacy, uptake of this strategy has been rather limited. US FDA guidelines advise discontinuation of doxorubicin in the event of significant objective deterioration of cardiac function.

\section{The unanticipated cardiotoxicity of 'targeted' therapies}

The accelerating pace of discovery brought numerous novel chemotherapeutic agents to market in the past decade. This new generation of therapies was developed to augment or replace a host of 'traditional' chemotherapeutic agents, such as anthracyclines, with variable efficacies and broad toxicities. Many of these targeted therapies have significantly more favorable safety and tolerability profiles; however, unanticipated adverse effects have been identified. One of the more salient and troubling adverse effects associated with multiple new chemotherapeutic agents is cardiotoxicity (Table 1). Over a decade ago, it became apparent that a surprising and significant number of patients were developing a cardiomyopathy associated with trastuzumab - the humanized monoclonal antibody targeting HER2 (also known as ErbB2) [39,40]. This unexpected complication detracts from the otherwise-impressive tolerability and exceptional efficacy of this agent, which meaningfully extends disease-free survival in women with HER2 ${ }^{+}$breast cancer $[39,41,42]$.

Although clinical trials report significantly lower incidence [41], it is likely that trastuzumab-associated cardiotoxicity occurs in roughly $20-33 \%$ of patients who receive the drug [12,43-45]. The resultant cardiomyopathy is often mild and reversible - particularly with increased dosing intervals between the anthracycline and trastuzumab - although its long-term implications on cardiovascular morbidity and mortality are unclear. Recent publication of NSABP B-31 clinical trial data demonstrate that the majority of the approximately $10 \%$ of subjects who developed trastuzumab-associated cardiotoxicity during the initial trial period had normal cardiac function at the 7-year follow-up [46]. However, longitudinal follow-up now suggests that the myocardial damage can be both severe and chronic $[43,45]$.

Importantly, the reaction appears to be idiosyncratic and independent of cumulative dose. The risk of developing cardiotoxicity is likely enhanced by the concomitant use of doxorubicin, and is more common in older patients with cardiac comorbidities [20,47]. The current approach for surveillance of cardiac damage is patterned after the approach used in anthracycline recipients, employing echocardiography and occasionally cardiac biomarkers. At present, there are no evidence-based therapies for trastuzumab-related cardiotoxicity, and its detection frequently leads to dose modification or even discontinuation of an otherwise highly effective treatment for breast cancer [46]. Given its impressive efficacy in the treatment of HER $2^{+}$breast cancer, trastuzumab will likely continue to be a cornerstone of adjuvant chemotherapy, underscoring the importance of both mechanistic study and further longitudinal cardiac outcomes data to guide prospective clinical decision-making. 
Trastuzumab-associated cardiotoxicity is incompletely understood but apparently does not lead to short-term loss of cardiomyocytes or significant ultrastructural myocardial abnormalities [48]. This, and other apparent distinctions, have led some to categorize trastuzumab-associated cardiotoxicity as a 'type II' effect, as opposed to the irreversible, 'type I' effect associated with anthracyclines [36]. The mechanism of trastuzumabassociated cardiotoxicity remains unclear, although its emergence gave rise to a biologically meaningful body of work that has described the importance of the neuregulin-1-ErbB2 signaling axis in the heart [49]. In many respects, the investigation of this signaling axis has generated a revised paradigm wherein the heart is now understood to be a functionally pluricellular organ, rather than a collection of cardiomyocytes structurally supported by fibroblasts. In this framework, cardiomyocyte receptors (e.g., ErbB2) participate in complex regulatory crosstalk with endothelial cells and fibroblasts via paracrine stimuli (e.g., neuregulin). This expanded recognition of the important roles of 'nonmyocytes' in the heart has broadly enhanced our understanding of myocardial biology and may also shed light on previously unrecognized mechanisms of drug-induced cardiotoxicity.

Multiple new chemotherapeutic agents selectively targeting tyrosine kinases have been introduced in recent years and their clinical applications have been expanding. It was hoped that the specificity of these small molecules - including imatinib, sunitinib, dasatinib and nilotinib - for a chosen tyrosine kinase would minimize adverse effects; however, sunitinib causes clinically apparent cardiotoxicity [50], and emerging data on other members of the class suggest a similar potential [51,52]. More recently it also has become evident that bevacizumab, a monoclonal antibody to VEGF-A, also is associated with cardiomyopathy and heart failure [53].

The myocardial damage induced by these ostensibly targeted therapies likely results from the cumulative response to both 'on-target' and 'off-target' effects. The on-target adverse events reflect the medical and scientific community's incomplete understanding of the biological roles of the target molecule. The off-target toxicities result from an incomplete characterization of the biological action of the drug itself. The efficacy of these compounds - and the lack of appealing extant therapeutic options - understandably brings them to market more quickly than drugs with other disease targets. The relatively brief period of time between development and clinical uptake emphasizes the critical importance of developing risk-stratification measures that will maximize therapeutic benefit and minimize both predictable and unpredictable harm. Achieving this balance requires both developing the 'right' drug, and selecting the appropriate patients to receive that drug. Recently, investigators introduced a novel preclinical strategy to predict tyrosine kinase inhibitor toxicity using zebrafish, and proposed that this clever approach would be useful in determining the cardiac toxicity of future drug candidates [54]. However, at present, our ability to prospectively identify patients at high risk for the development of chemotherapeutic cardiotoxicity remains limited.

\section{Risk stratification: clinical \& demographic profiling}

The likelihood of developing cardiomyopathy secondary to doxorubicin is increased by higher cumulative dose [8], concomitant irradiation [55], cardiovascular comorbidities [56], age $>65$ years [10] and female sex [57]. Advanced age and cardiovascular comorbidities also appear to increase the likelihood of developing trastuzumab-associated cardiotoxicity [58], and concomitant administration of doxorubicin clearly increases risk [39]. Sunitinibassociated cardiotoxicity also appears to occur with greater frequency in older patients with pre-existing cardiac disease [50]. Such demographic profiles are blunt instruments for risk assessment, and it would be both impractical and medically disadvantageous to limit the use of these highly efficacious chemotherapeutic agents to young patients without comorbidities. 
Given the ever-expanding population of patients with both cardiac risk factors and cancer, there is a pressing need for more sophisticated tools to stratify risk for chemotherapeutic cardiotoxicity.

Importantly, a significant number of adverse effects occur in patients without obvious risk factors. For example, some patients develop profound anthracycline-induced cardiotoxicity at low doses of doxorubicin, whereas other patients tolerate very high doses without any myocardial damage [59]. There is no clear relationship between cumulative dose of trastuzumab and the likelihood of developing cardiotoxicity [43], and the adverse cardiac effects of tyrosine kinase inhibitors are likely to be similarly idiosyncratic [60]. The incomplete prediction of adverse response by demographic and clinical factors bespeaks a predisposition to susceptibility that may well be genetically determined. Pharmacogenetic profiling of chemotherapeutic cardiotoxicity has to date been limited, but holds promise as a means to maximize anticancer benefit and minimize cardiotoxic harm.

\section{Risk stratification: candidate-gene approaches}

Extant knowledge of the genetic determinants of susceptibility to the cardiotoxicity of trastuzumab and other targeted therapies is limited. Candidate-gene approaches naturally have focused on variants in the molecular targets for these drugs, although this approach clearly underestimates their mechanistic complexity. One small study suggested that an $E r b B 2$ polymorphism (Ile655Val) conferred both increased growth potential in tumor cells and an increased propensity for cardiotoxicity in patients treated with trastuzumab [61]. However, it is unlikely that a single, common variant is sufficient to explain the risk of this untoward effect.

Numerous investigators have employed a candidate-gene approach to genotyping risk for doxorubicin-induced cardiotoxicity. In each case, the selection of SNPs has been guided by the presumed mechanism of myocardial injury, by knowledge of the pathways responsible for doxorubicin trafficking and elimination, or by demonstration of inducible protection against anthracycline-induced cardiotoxicity (Figure 1) [62]. Although this approach has met with some success, it inherently is limited by the incomplete understanding of the mechanisms underlying the myocardial insult.

Animal studies have suggested critical roles for numerous genes involved in oxidative stress, including Nox2 [22], NOS2a, MnSOD [23], PTGS2 [24], MT [25] and TRX1 [26]. Genes involved in the metabolism of doxorubicin also have been implicated in mouse models, such as $C B R 1, C B R 3$ [63] and $A B C B 1 / M D R 1$ [64]. Transgenic mice also have helped to identify the roles of STAT3 [29] and GATA4 [27,28] in the transcriptional regulation of the response to doxorubicin-induced myocardial injury. Although such studies may provide some insight regarding the effect of dramatic alterations in the abundance or function of a given protein, they rarely predict a response in the human system. Among the variety of reasons for this discordance, perhaps the most important is the fact that human gene variants rarely result in either the complete failure of protein expression (as in a knockout mouse) or the supraphysiologic levels of overexpression seen in many transgenic mouse models. Thus, candidate-gene approaches to identify determinants of chemotherapeutic cardiotoxicity have not been particularly fruitful, with a few noteworthy exceptions.

One such study evaluated SNPs from 82 genes with a 'conceivable role in anthracycline cardiotoxicity' [65]. The authors selected candidate-gene variants based on their role in reactive oxygen species generation, doxorubicin transport or metabolism, and in the development of heart failure due to other causes. Subjects were adults (mean age: 62 years) drawn from the German High-Grade Non-Hodgkin Lymphoma Study Group who developed either chronic or acute cardiotoxicity after a cumulative doxorubicin dose of $300 \mathrm{mg} / \mathrm{m}^{2}$. 
The authors identified five variants in genes encoding either NADPH oxidase subunits or doxorubicin efflux transporter ABCB1/MDR1 that conveyed up to 2.5-fold increased risk for cardiotoxicity.

A subsequent study from a separate group confirmed an increased susceptibility to doxorubicin-induced cardiotoxicity in patients with the $-212 \mathrm{~A}>\mathrm{G}$ variant of the gene encoding the NCF4 subunit of NADPH oxidase [66]. Smaller candidate-gene studies have implicated variants in other genes encoding parts of the doxorubicin metabolism pathway ( $C B R 1$ and $C B R 3$ [67], as well as $A B C C 1$ [68]). However, others failed to find an increased risk associated with variants in numerous genes involved in oxidative homeostasis [69].

More recently, Visscher et al. published the largest effort to date investigating genetic determinants of doxorubicin-induced cardiotoxicity on behalf of the Canadian Pharmacogenomics Network for Drug Safety Consortium [70]. The authors studied 2977 SNPs in 220 drug biotransformation genes in a discovery cohort and two separate replication cohorts in Canada and The Netherlands. The subjects were children who received anthracyclines for the treatment of various malignancies and developed cardiotoxicity during at least 5 years of follow-up. A synonymous coding variant in the solute $S L C 28 A 3$ or $h C N T 3$ gene conferred significant protection (odds ratio: 0.31) against doxorubicin-induced cardiotoxicity. Multiple variants in $A B C B 1, A B C B 4$ and $A B C C 1$ also were found to be protective. Based on these results, the investigators developed a multigene risk-stratification tool and retrospectively used it to group the discovery and replication cohorts in tertiles of risk. The positive predictive value for classification as high risk was $75 \%$ and the negative predictive value for low-risk classification was $96 \%$.

Interestingly, this study failed to confirm the previously published association between susceptibi lity to doxorubicin-induced cardiotoxicity and variants in $A B C C 1$ and NCF4 $[65,66]$. The discrepancy may arise from the fact that the previous studies analyzed SNPs from adult patients, whereas Visscher et al. assayed a pediatric population. The differences between adult and pediatric susceptibility to doxorubicin have been recognized for over four decades [7] and likely limit the applicability of these findings to the adult population. There are also important ascertainment differences, with Visscher et al. accruing from referral centers while some of the other studies obtained patients as part of clinical trials. The observed differences may also simply reflect the inherent statistical shortcomings of studying a limited patient sample. Although clearly not ready for clinical application, these noteworthy findings provide a glimpse into the potential predictive power of using genetic analysis to prospectively predict risk of cardiotoxicity in patients who may receive anthracycline-based chemotherapy.

\section{Toward a pharmacogenomic determination of risk}

The power of the candidate-gene approach to predict a given genotype-phenotype relationship is directly proportional to the degree to which the mechanistic determinants of that phenotype have been identified. Our understanding of the mechanisms underlying the cardiomyopathy that complicates trastuzumab and the tyrosine kinase inhibitors is likely too narrow to inform effective candidate-gene efforts at risk profiling, although great strides are being made in this area $[60,71]$.

Conventional scientific wisdom has long held that doxorubicin-induced cardiotoxicity is mediated largely by cell death in the setting of oxidative stress; however, the shortcomings of that paradigm are becoming increasingly clear [19]. Newer technologies permitting higher-throughput analysis have identified contributions from pathways that have not previously been investigated. Proteomic analysis of the left ventricle of rabbits chronically exposed to doxorubicin reveal distinct alterations in the mitochondrial metabolic signature, 
as well as altered abundance of key cytoskeletal and sarcomeric proteins in the setting of activation of the proteasome system [72]. A genome-wide screen in doxorubicin-treated yeast [73] and whole-genome linkage scans in human lymphoblastoid cell lines [74] have also offered new glimpses into the complexity of the genetic determination of susceptibility to doxorubicin-induced cytotoxicity. Whether these findings apply to human myocardial biology remains to be seen, as there have been no large-scale efforts to query the human genome for markers of risk for chemotherapeutic cardiotoxicity.

Genetic testing already guides dosing of multiple antineoplastic agents [75], and chemotherapeutic cardiotoxicity seems a very promising target for broader pharmacogenomic assessment. The adverse drug effect is highly morbid and is likely to increase in frequency and severity as the number of patients with both heart disease and cancer increases. The unpredictable and serious nature of doxorubicin-induced cardiotoxicity has led to underuse of anthracyclines [76] and consideration of abandoning this highly effective chemotherapeutic class altogether [77]. Indeed it is not hyperbolic to observe that both the disease and the treatment itself carry life-threatening risk. There is clear variability in susceptibility to myocardial damage that is incompletely explained by clinical and demographic factors, and early evidence demonstrates genetic predisposition. The identification of this complication prompts the discontinuation of otherwise effective treatment, thus enhanced risk stratification could both minimize harm and maximize benefit. Broader exploration for the genetic determinants of risk for chemotherapeutic cardiotoxicity may even shed further light on the mechanisms underlying this unanticipated and highly morbid adverse drug effect.

\section{Future perspective}

Chemotherapeutic cardiotoxicity appears ripe for further investigation. Indeed, a subspecialty has recently been created for the study of this and related problems, such as 'cardio-oncology'. Expanded preclinical molecular research will enhance our understanding of the underlying mechanisms and novel nonhuman genetic resources will provide opportunities for broader genomic analysis. Given the large volume of patients who receive these medications and the relative frequency with which cardiotoxicity occurs, it seems likely that human genome-wide association studies will be undertaken within the next decade. Synthesis of these various approaches will undoubtedly lead to a deeper understanding of the genetic determinants of chemotherapeutic cardiotoxicity. If such studies identify variants that confer meaningful predisposition to cardiotoxicity, genetic screening could be integrated with established clinical and demographic factors to mitigate risk of developing this highly morbid adverse effect.

\section{References}

Papers of special note have been highlighted as:

- of interest

- of considerable interest

1. Tewey KM, Chen GL, Nelson EM, Liu LF. Intercalative antitumor drugs interfere with the breakage-reunion reaction of mammalian DNA topoisomerase II. J Biol Chem. 1984; 259(14): 9182-9187. [PubMed: 6086625]

2. Arcamone F, Cassinelli G, Fantini G, et al. Adriamycin, 14-hydroxydaunomycin, a new antitumor antibiotic from $S$. peucetius var.caesius. Biotechnol Bioeng. 1969; 11(6):1101-1110. [PubMed: 5365804] 
3. Blum RH, Carter SK. Adriamycin. A new anticancer drug with significant clinical activity. Ann Intern Med. 1974; 80(2):249-259. [PubMed: 4590654]

4. Early Breast Cancer Trialists' Collaborative Group (EBCTCG). Effects of chemotherapy and hormonal therapy for early breast cancer on recurrence and 15-year survival: an overview of the randomised trials. Lancet. 2005; 365(9472):1687-1717. [PubMed: 15894097]

5. Tan C, Tasaka H, Yu KP, Murphy ML, Karnofsky DA. Daunomycin, an antitumor antibiotic, in the treatment of neoplastic disease. Clinical evaluation with special reference to childhood leukemia. Cancer. 1967; 20(3):333-353. [PubMed: 4290058]

6. Lefrak EA, Pitha J, Rosenheim S, Gottlieb JA. A clinicopathologic analysis of adriamycin cardiotoxicity. Cancer. 1973; 32(2):302-314. [PubMed: 4353012]

7. Bonadonna G, Monfardini S. Cardiac toxicity of daunorubicin. Lancet. 1969; 1(7599):837. [PubMed: 4180385]

8m. Von Hoff DD, Layard MW, Basa P, et al. Risk factors for doxorubicin-induced congestive heart failure. Ann Intern Med. 1979; 91(5):710-717. Most comprehensive retrospective analysis of clinical risk factors for anthracycline-induced cardiotoxicity. [PubMed: 496103]

9. Lipshultz SE, Lipsitz SR, Sallan SE, et al. Chronic progressive cardiac dysfunction years after doxorubicin therapy for childhood acute lymphoblastic leukemia. J Clin Oncol. 2005; 23(12):26292636. [PubMed: 15837978]

10. Swain SM, Whaley FS, Ewer MS. Congestive heart failure in patients treated with doxorubicin: a retrospective analysis of three trials. Cancer. 2003; 97(11):2869-2879. [PubMed: 12767102]

11. Kremer LC, Van Dalen EC, Offringa M, Voute PA. Frequency and risk factors of anthracyclineinduced clinical heart failure in children: a systematic review. Ann Oncol. 2002; 13(4):503-512. [PubMed: 12056699]

12. Verma S, Ewer MS. Is cardiotoxicity being adequately assessed in current trials of cytotoxic and targeted agents in breast cancer? Ann Oncol. 2010; 22(5):1011-1018. [PubMed: 21097988]

13. Kremer LC, van der Pal HJ, Offringa M, van Dalen EC, Voute PA. Frequency and risk factors of subclinical cardiotoxicity after anthracycline therapy in children: a systematic review. Ann Oncol. 2002; 13(6):819-829. [PubMed: 12123328]

14. Wang TJ, Evans JC, Benjamin EJ, Levy D, Leroy EC, Vasan RS. Natural history of asymptomatic left ventricular systolic dysfunction in the community. Circulation. 2003; 108(8):977-982. [PubMed: 12912813]

15. Kopp LM, Bernstein ML, Schwartz CL, et al. The effects of dexrazoxane on cardiac status and second malignant neoplasms $(\mathrm{SMN})$ in doxorubicin-treated patients with osteosarcoma (OS). $\mathrm{J}$ Clin Oncol. 2012; 30(Suppl):Abstract 9503.

16. Landy DC, Miller TL, Lopez-Mitnik G, et al. Aggregating traditional cardiovascular disease risk factors to assess the cardiometabolic health of childhood cancer survivors: an analysis from the Cardiac Risk Factors in Childhood Cancer Survivors Study. Am Heart J. 2012; 163(2):295-301. e292. [PubMed: 22305850]

17. Lipshultz SE, Landy DC, Lopez-Mitnik G, et al. Cardiovascular status of childhood cancer survivors exposed and unexposed to cardiotoxic therapy. J Clin Oncol. 2012; 30(10):1050-1057. [PubMed: 22393080]

18. Pinder MC, Duan Z, Goodwin JS, Hortobagyi GN, Giordano SH. Congestive heart failure in older women treated with adjuvant anthracycline chemotherapy for breast cancer. J Clin Oncol. 2007; 25(25):3808-3815. [PubMed: 17664460]

19m. Gianni L, Herman EH, Lipshultz SE, Minotti G, Sarvazyan N, Sawyer DB. Anthracycline cardiotoxicity: from bench to bedside. J Clin Oncol. 2008; 26(22):3777-3784. Summary statement from the First International Workshop on Anthracycline Cardiotoxicity providing an excellent summary of extant knowledge on anthracycline-induced cardiotoxicity and identifying important future directions for translation of that knowledge to clinical benefit. [PubMed: 18669466]

20. Raschi E, Vasina V, Ursino MG, Boriani G, Martoni A, De Ponti F. Anticancer drugs and cardiotoxicity: insights and perspectives in the era of targeted therapy. Pharmacol Ther. 2010; 125(2):196-218. [PubMed: 19874849] 
21. Chen B, Peng X, Pentassuglia L, Lim CC, Sawyer DB. Molecular and cellular mechanisms of anthracycline cardiotoxicity. Cardiovasc Toxicol. 2007; 7(2):114-121. [PubMed: 17652815]

22. Zhao Y, Mclaughlin D, Robinson E, et al. Nox2 NADPH oxidase promotes pathologic cardiac remodeling associated with doxorubicin chemotherapy. Cancer Res. 2010; 70(22):9287-9297. [PubMed: 20884632]

23. Cole MP, Chaiswing L, Oberley TD, et al. The protective roles of nitric oxide and superoxide dismutase in adriamycin-induced cardiotoxicity. Cardiovasc Res. 2006; 69(1):186-197. [PubMed: 16157314]

24. Dowd NP, Scully M, Adderley SR, Cunningham AJ, Fitzgerald DJ. Inhibition of cyclooxygenase-2 aggravates doxorubicin-mediated cardiac injury in vivo. J Clin Invest. 2001; 108(4):585-590. [PubMed: 11518732]

25. Kang YJ, Chen Y, Yu A, Voss-McCowan M, Epstein PN. Overexpression of metallothionein in the heart of transgenic mice suppresses doxorubicin cardiotoxicity. J Clin Invest. 1997; 100(6): 1501-1506. [PubMed: 9294117]

26. Shioji K, Kishimoto C, Nakamura H, et al. Overexpression of thioredoxin-1 in transgenic mice attenuates adriamycin-induced cardiotoxicity. Circulation. 2002; 106(11):1403-1409. [PubMed: 12221060]

27. Aries A, Paradis P, Lefebvre C, Schwartz RJ, Nemer M. Essential role of GATA-4 in cell survival and drug-induced cardiotoxicity. Proc Natl Acad Sci USA. 2004; 101(18):6975-6980. [PubMed: 15100413]

28. Kim Y, Ma AG, Kitta K, et al. Anthracycline-induced suppression of GATA-4 transcription factor: implication in the regulation of cardiac myocyte apoptosis. Mol Pharmacol. 2003; 63(2):368-377. [PubMed: 12527808]

29. Kunisada K, Negoro S, Tone E, et al. Signal transducer and activator of transcription 3 in the heart transduces not only a hypertrophic signal but a protective signal against doxorubicin-induced cardiomyopathy. Proc Natl Acad Sci USA. 2000; 97(1):315-319. [PubMed: 10618415]

30. Lien YC, Noel T, Liu H, Stromberg AJ, Chen KC, St Clair DK. Phospholipase C-delta1 is a critical target for tumor necrosis factor receptor-mediated protection against adriamycin-induced cardiac injury. Cancer Res. 2006; 66(8):4329-4338. [PubMed: 16618758]

31. Niu J, Azfer A, Wang K, Wang X, Kolattukudy PE. Cardiac-targeted expression of soluble fas attenuates doxorubicin-induced cardiotoxicity in mice. J Pharmacol Exp Ther. 2009; 328(3):740748. [PubMed: 19066339]

32. Yoshida M, Shiojima I, Ikeda H, Komuro I. Chronic doxorubicin cardiotoxicity is mediated by oxidative DNA damage-ATM-p53-apoptosis pathway and attenuated by pitavastatin through the inhibition of Rac1 activity. J Mol Cell Cardiol. 2009; 47(5):698-705. [PubMed: 19660469]

33. Speyer JL, Green MD, Kramer E, et al. Protective effect of the bispiperazinedione ICRF 187 against doxorubicin-induced cardiac toxicity in women with advanced breast cancer. $\mathrm{N}$ Engl J Med. 1988; 319(12):745-752. [PubMed: 3137469]

34. Marty M, Espie M, Llombart A, Monnier A, Rapoport BL, Stahalova V. Multicenter randomized Phase III study of the cardioprotective effect of dexrazoxane (cardioxane) in advanced/metastatic breast cancer patients treated with anthracycline-based chemotherapy. Ann Oncol. 2006; 17(4): 614-622. [PubMed: 16423847]

35. Miranda CJ, Makui H, Soares RJ, et al. Hfe deficiency increases susceptibility to cardiotoxicity and exacerbates changes in iron metabolism induced by doxorubicin. Blood. 2003; 102(7):25742580. [PubMed: 12805055]

36. Ewer MS, Ewer SM. Cardiotoxicity of anticancer treatments: what the cardiologist needs to know. Nat Rev Cardiol. 2010; 7(10):564-575. Straightforward review written expressly for cardiologists who will care for patients who develop cardiomyopathy due to antineoplastic agents. [PubMed: 20842180]

37. Lipshultz SE, Miller TL, Scully RE, et al. Changes in cardiac biomarkers during doxorubicin treatment of pediatric patients with high-risk acute lymphoblastic leukemia: associations with long-term echocardiographic outcomes. J Clin Oncol. 2012; 30(10):1042-1049. [PubMed: 22370326] 
38. Yoon GJ, Telli ML, Kao DP, Matsuda KY, Carlson RW, Witteles RM. Left ventricular dysfunction in patients receiving cardiotoxic cancer therapies are clinicians responding optimally? J Am Coll Cardiol. 2010; 56(20):1644-1650. [PubMed: 21050974]

39. Slamon DJ, Leyland-Jones B, Shak S, et al. Use of chemotherapy plus a monoclonal antibody against HER2 for metastatic breast cancer that overexpresses HER2. N Engl J Med. 2001; 344(11):783-792. [PubMed: 11248153]

40. Feldman AM, Lorell BH, Reis SE. Trastuzumab in the treatment of metastatic breast cancer: anticancer therapy versus cardiotoxicity. Circulation. 2000; 102(3):272-274. [PubMed: 10899087]

41. Piccart-Gebhart MJ, Procter M, Leyland-Jones B, et al. Trastuzumab after adjuvant chemotherapy in HER2-positive breast cancer. N Engl J Med. 2005; 353(16):1659-1672. [PubMed: 16236737]

42. Slamon D, Eiermann W, Robert N, et al. Adjuvant trastuzumab in HER2-positive breast cancer. N Engl J Med. 2011; 365(14):1273-1283. [PubMed: 21991949]

43. Guglin M, Hartlage G, Reynolds C, Chen R, Patel V. Trastuzumab-induced cardiomyopathy: not as benign as it looks? A retrospective study. J Card Fail. 2009; 15(8):651-657. [PubMed: 19786253]

44- Tarantini L, Cioffi G, Gori S, et al. Trastuzumab adjuvant chemotherapy and cardiotoxicity in real-world women with breast cancer. J Card Fail. 2012; 18(2):113-119. Retrospective analysis of trastuzumab-associated cardiotoxicity revealing a real-world incidence that is significantly higher than reported in many clinical trials. [PubMed: 22300778]

45. Telli ML, Hunt SA, Carlson RW, Guardino AE. Trastuzumab-related cardiotoxicity: calling into question the concept of reversibility. J Clin Oncol. 2007; 25(23):3525-3533. [PubMed: 17687157]

46. Romond EH, Jeong JH, Rastogi P, et al. Seven-year follow-up assessment of cardiac function in NSABP B-31, a randomized trial comparing doxorubicin and cyclophosphamide followed by paclitaxel (ACP) with ACP plus trastuzumab as adjuvant therapy for patients with node-positive, human epidermal growth factor receptor 2-positive breast cancer. J Clin Oncol. 2012; 30(31): 3792-3799. [PubMed: 22987084]

47. Chen J, Long JB, Hurria A, Owusu C, Steingart RM, Gross CP. Incidence of heart failure or cardiomyopathy after adjuvant trastuzumab therapy for breast cancer. J Am Coll Cardiol. 2012; 60(24):2504-2512. [PubMed: 23158536]

48. Ewer MS, Vooletich MT, Durand JB, et al. Reversibility of trastuzumab-related cardiotoxicity: new insights based on clinical course and response to medical treatment. J Clin Oncol. 2005; 23(31):7820-7826. [PubMed: 16258084]

49. Lemmens K, Doggen K, De Keulenaer GW. Role of neuregulin-1/ErbB signaling in cardiovascular physiology and disease: implications for therapy of heart failure. Circulation. 2007; 116(8):954960. [PubMed: 17709650]

50. Chu TF, Rupnick MA, Kerkela R, et al. Cardiotoxicity associated with tyrosine kinase inhibitor sunitinib. Lancet. 2007; 370(9604):2011-2019. [PubMed: 18083403]

51. Force T, Krause DS, Van Etten RA. Molecular mechanisms of cardiotoxicity of tyrosine kinase inhibition. Nat Rev Cancer. 2007; 7(5):332-344. [PubMed: 17457301]

52. Mellor HR, Bell AR, Valentin JP, Roberts RR. Cardiotoxicity associated with targeting kinase pathways in cancer. Toxicol Sci. 2011; 120(1):14-32. [PubMed: 21177772]

53. Mcgonigle KF, Muntz HG, Vuky J, et al. Combined weekly topotecan and biweekly bevacizumab in women with platinum-resistant ovarian, peritoneal, or fallopian tube cancer: results of a Phase 2 study. Cancer. 2011; 117(16):3731-3740. [PubMed: 21815133]

54. Cheng H, Kari G, Dicker AP, Rodeck U, Koch WJ, Force T. A novel preclinical strategy for identifying cardiotoxic kinase inhibitors and mechanisms of cardiotoxicity. Circ Res. 2011; 109(12):1401-1409. [PubMed: 21998323]

55. Steinherz LJ, Steinherz PG, Tan CT, Heller G, Murphy ML. Cardiac toxicity 4 to 20 years after completing anthracycline therapy. JAMA. 1991; 266(12):1672-1677. [PubMed: 1886191]

56. Hershman DL, Mcbride RB, Eisenberger A, Tsai WY, Grann VR, Jacobson JS. Doxorubicin, cardiac risk factors, and cardiac toxicity in elderly patients with diffuse B-cell non-Hodgkin's lymphoma. J Clin Oncol. 2008; 26(19):3159-3165. [PubMed: 18591554] 
57. Lipshultz SE, Lipsitz SR, Mone SM, et al. Female sex and drug dose as risk factors for late cardiotoxic effects of doxorubicin therapy for childhood cancer. N Engl J Med. 1995; 332(26): 1738-1743. [PubMed: 7760889]

58. Serrano C, Cortes J, De Mattos-Arruda L, et al. Trastuzumab-related cardiotoxicity in the elderly: a role for cardiovascular risk factors. Ann Oncol. 2012; 23(4):897-902. [PubMed: 21828361]

59. Henderson IC, Allegra JC, Woodcock T, et al. Randomized clinical trial comparing mitoxantrone with doxorubicin in previously treated patients with metastatic breast cancer. J Clin Oncol. 1989; 7(5):560-571. [PubMed: 2468745]

60". Force T, Kolaja KL. Cardiotoxicity of kinase inhibitors: the prediction and translation of preclinical models to clinical outcomes. Nat Rev Drug Discov. 2011; 10(2):111-126. Excellent review of the cardiotoxicity of novel 'targeted' antineoplastic agents from the leading experts in the field. [PubMed: 21283106]

61. Beauclair S, Formento P, Fischel JL, et al. Role of the HER2 [Ile655Val] genetic polymorphism in tumorogenesis and in the risk of trastuzumab-related cardiotoxicity. Ann Oncol. 2007; 18(8): 1335-1341. [PubMed: 17693647]

62. Jensen BC, O’Connell TD, Simpson PC. Alpha-1-adrenergic receptors: targets for agonist drugs to treat heart failure. J Mol Cell Cardiol. 2011; 51(4):518-528. [PubMed: 21118696]

63. Forrest GL, Gonzalez B, Tseng W, Li X, Mann J. Human carbonyl reductase overexpression in the heart advances the development of doxorubicin-induced cardiotoxicity in transgenic mice. Cancer Res. 2000; 60(18):5158-5164. [PubMed: 11016643]

64. Dell'acqua G, Polishchuck R, Fallon JT, Gordon JW. Cardiac resistance to adriamycin in transgenic mice expressing a rat alpha-cardiac myosin heavy chain/human multiple drug resistance 1 fusion gene. Hum Gene Ther. 1999; 10(8):1269-1279. [PubMed: 10365658]

65-. Wojnowski L, Kulle B, Schirmer M, et al. NAD(P)H oxidase and multidrug resistance protein genetic polymorphisms are associated with doxorubicin-induced cardiotoxicity. Circulation. 2005; 112(24):3754-3762. Used a candidate-gene approach to identify multiple SNPs that confer increased risk for anthracycline-induced cardiotoxicity in adults. [PubMed: 16330681]

66. Rossi D, Rasi S, Franceschetti S, et al. Analysis of the host pharmacogenetic background for prediction of outcome and toxicity in diffuse large B-cell lymphoma treated with R-CHOP21. Leukemia. 2009; 23(6):1118-1126. [PubMed: 19448608]

67. Blanco JG, Leisenring WM, Gonzalez-Covarrubias VM, et al. Genetic polymorphisms in the carbonyl reductase 3 gene $C B R 3$ and the $\mathrm{NAD}(\mathrm{P}) \mathrm{H}$ :quinone oxidoreductase 1 gene $N Q O 1$ in patients who developed anthracycline-related congestive heart failure after childhood cancer. Cancer. 2008; 112(12):2789-2795. [PubMed: 18457324]

68. Semsei AF, Erdelyi DJ, Ungvari I, et al. $A B C C 1$ polymorphisms in anthracycline-induced cardiotoxicity in childhood acute lymphoblastic leukaemia. Cell Biol Int. 2012; 36(1):79-86. [PubMed: 21929509]

69. Rajic V, Aplenc R, Debeljak M, et al. Influence of the polymorphism in candidate genes on late cardiac damage in patients treated due to acute leukemia in childhood. Leuk Lymphoma. 2009; 50(10):1693-1698. [PubMed: 19863340]

70m. Visscher H, Ross CJ, Rassekh SR, et al. Pharmacogenomic prediction of anthracycline-induced cardiotoxicity in children. J Clin Oncol. 2011; 30(13):1422-1428. Most extensive pharmacogenetic analysis of potential genetic determinants of anthracycline-induced cardiotoxicity conducted to date. [PubMed: 21900104]

71. Zambelli A, Della Porta MG, Eleuteri E, et al. Predicting and preventing cardiotoxicity in the era of breast cancer targeted therapies. Novel molecular tools for clinical issues. Breast. 2010; 20(2): 176-183. [PubMed: 21146409]

72. Sterba M, Popelova O, Lenco J, et al. Proteomic insights into chronic anthracycline cardiotoxicity. J Mol Cell Cardiol. 2011; 50(5):849-862. [PubMed: 21284945]

73. Xia L, Jaafar L, Cashikar A, Flores-Rozas H. Identification of genes required for protection from doxorubicin by a genome-wide screen in Saccharomyces cerevisiae. Cancer Res. 2007; 67(23): 11411-11418. [PubMed: 18056469]

74. Duan S, Bleibel WK, Huang RS, et al. Mapping genes that contribute to daunorubicin-induced cytotoxicity. Cancer Res. 2007; 67(11):5425-5433. [PubMed: 17545624] 
75. Wang L, McLeod HL, Weinshilboum RM. Genomics and drug response. N Engl J Med. 2011; 364(12):1144-1153. [PubMed: 21428770]

76. Montemurro F, Rossi V, Nole F, et al. Underuse of anthracyclines in women with HER-2 ${ }^{+}$ advanced breast cancer. Oncologist. 2010; 15(7):665-672. [PubMed: 20576644]

77. Jones SE, Savin MA, Holmes FA, et al. Phase III trial comparing doxorubicin plus cyclophosphamide with docetaxel plus cyclophosphamide as adjuvant therapy for operable breast cancer. J Clin Oncol. 2006; 24(34):5381-5387. [PubMed: 17135639]

78. Choueiri TK, Mayer EL, Je Y, et al. Congestive heart failure risk in patients with breast cancer treated with bevacizumab. J Clin Oncol. 2011; 29(6):632-638. [PubMed: 21205755] 


\section{Executive summary}

\section{Anthracyclines: the past \& present of chemotherapeutic cardiotoxicity}

- The anthracycline antibiotics, exemplified by doxorubicin (adriamycin), have broad antineoplastic efficacy, but are associated with damage to the heart (cardiotoxicity) that can be severe and permanent.

- Anthracycline-induced cardiotoxicity is partially dependent on cumulative dose, but may occur at lower doses in patients with cardiac comorbidities or idiosyncratically in patients with no obvious risk factors. In this article, we present evidence that indicates a genetic predisposition to this important adverse drug effect.

- Oxidative stress and apoptosis contribute to cardiomyocyte death, but the mechanisms underlying anthracycline-induced cardiotoxicity are incompletely understood.

\section{The unanticipated cardiotoxicity of targeted therapies}

- Newer antineoplastic agents with specific molecular targets also cause cardiotoxicity. The best known of these drugs is trastuzumab, the humanized monoclonal antibody to HER2 (also known as ErbB2).

- Multiple new drugs targeting tyrosine kinases also cause myocardial damage.

- The cardiotoxicity of targeted therapies results from both on-target and offtarget effects.

- Unlike anthracycline-induced cardiotoxicity, the cardiotoxicity of these agents is entirely idiosyncratic and is often reversible. Older patients and those with cardiac comorbidities are at greater risk for developing this adverse reaction.

\section{Risk stratification: clinical \& demographic profiling}

- The likelihood of developing cardiotoxicity due to anthracyclines increases with cumulative drug dose, concomitant radiation, age, sex and cardiovascular comorbidities.

- Trastuzumab-induced cardiotoxicity appears to be independent of cumulative dose, but occurs more often with concomitant doxorubicin administration and cardiovascular comorbidities.

\section{Risk stratification: candidate-gene approaches}

- Variants in the genes encoding NADPH oxidase subunits and doxorubicin efflux transporter $A B C B 1 / M D R 1$ were found to increase risk for anthracyclineinduced cardiotoxicity in adults.

- A variant in the $S L C 28 A 3$ gene conferred significant protection against anthracycline-induced cardiotoxicity in a large candidate-gene study of Canadian children. The investigators identified other protective variants and developed a pediatric multigene risk prediction model.

\section{Toward a pharmacogenomic determination of risk}

- Candidate-gene approaches are limited by our incomplete understanding of the mechanisms underlying chemotherapeutic cardiotoxicity. New proteomic and genomic analyses of nonhuman organisms indicate the complexity of the genetic determinants of this important adverse drug effect. 
- New ways to prospectively predict risk of chemotherapeutic cardiotoxicity are needed. Pharmacogenomic approaches hold promise for minimizing cardiotoxic harm and maximizing antineoplastic benefit associated with the use of chemotherapeutic agents. 


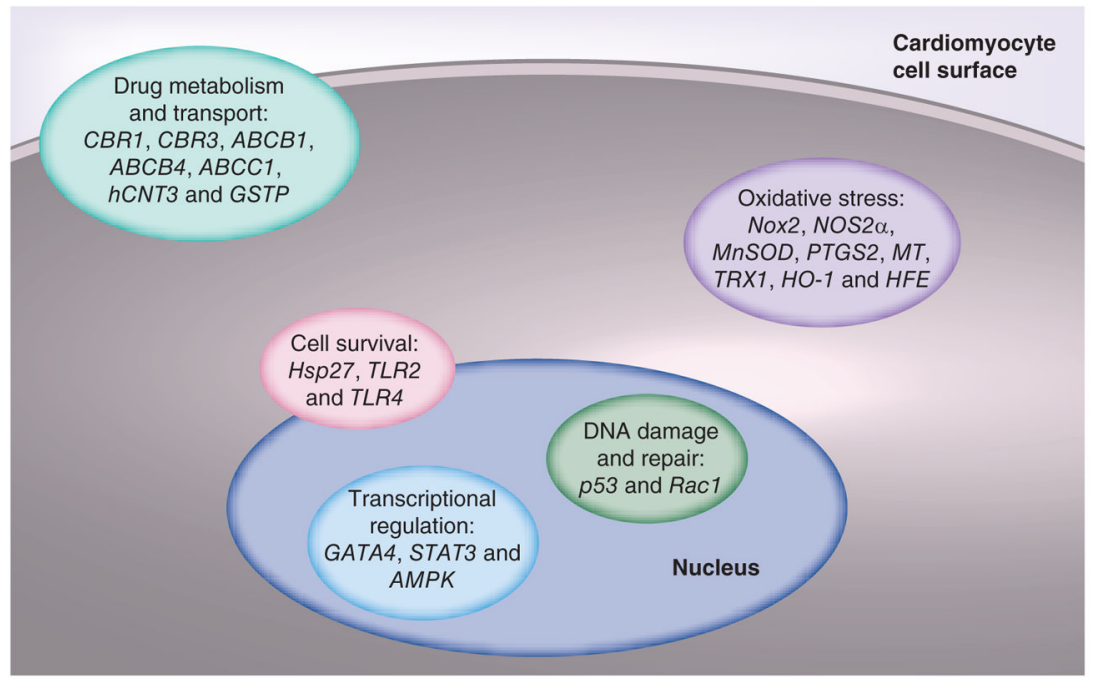

Figure 1. Candidate genes for susceptibility to anthracycline-induced cardiotoxicity Animal studies and limited human genetic analyses have identified candidate genes involved in a limited number of processes that are central to the pathogenesis of anthracyclineinduced cardiotoxicity. 
Table 1

Antineoplastic agents associated with cardiotoxicity.

\begin{tabular}{|llr|}
\hline Antineoplastic agent & Estimated prevalence of cardiotoxicity $(\%)$ & Ref. \\
\hline Adriamycin & $5-15$ (heart failure); 50 (subclinical cardiotoxicity) & {$[10,11,13]$} \\
\hline Trastuzumab & $20-33$ & {$[43-45]$} \\
\hline Sunitinib & $8-15$ (heart failure or cardiomyopathy) & {$[52]$} \\
\hline Dasatinib & 4 (heart failure or cardiomyopathy) & {$[52]$} \\
\hline Sorafenib & $3-17$ & {$[52]$} \\
\hline Imatinib & $0.5-1.7$ & {$[52]$} \\
\hline Bevacizumab & 1.6 & {$[78]$} \\
\hline
\end{tabular}

